\title{
Decline of Influenza Mortality in Canada since the Spanish Flu
}

\author{
Anthony A. Noce \\ School of Graduate Studies, Concordia University \\ 1455 De Maisonneuve Blvd. West, Montreal H3G 1M8, Canada \\ E-mail: anoce@alcor.concordia.ca
}

Michael C. Otterstatter

Applied Research and Analysis Directorate, Strategic Policy Branch, Health Canada

200 Eglantine Driveway, Ottawa K1A 0K9, Canada

E-mail: michael.otterstatter@phac-aspc.gc.ca

Zachary Jacobson (Corresponding author)

Applied Research and Analysis Directorate, Strategic Policy Branch, Health Canada

200 Eglantine Driveway, Ottawa K1A 0K9, Canada

Tel: 1-613-946-9056Ｅ-mail: zachary_jacobson@hc-sc.gc.ca

Equal contributions

Received: February 21, 2011 Accepted: March 7, 2011 doi:10.5539/gjhs.v3n2p29

This study was supported in part by the Health Canada Postdoctoral Fellowship program (M.C.O).

The views expressed in this article are not, in part or in whole, a reflection of those held by Health Canada or Concordia University, but strictly those of the authors.

\begin{abstract}
Influenza has long been an important source of mortality worldwide. However, the historical patterns of death due to this disease are still poorly understood. We present the first analysis of the long-term patterns of annual influenza mortality in Canada, covering nearly the entire period since the Spanish flu pandemic of 1918. The death rate due to influenza showed a clear exponential decay from 1922 (the earliest year accurate statistics are available) until 2003. In addition, a log transformation of the influenza specific death rate data revealed a rise in influenza mortality above the long-term trend during 1998-2000. There was no strong evidence of periodicity in influenza mortality, with the time series showing only weak positive autocorrelation. We compare the decline in influenza mortality to historical patterns in death due to pneumonia and other infectious respiratory diseases. The observed decay in influenza deaths could have been due, in part, to improvements in population health, public health practice, and the treatment of infectious diseases. However, we also argue that such a decline may have been due to improved, long-lasting, individual- and population-level immunity to influenza.
\end{abstract}

Keywords: Influenza, Mortality, Policy, Vaccination

\section{Introduction}

\subsection{Long term trends in influenza--significance}

During the 1918 'Spanish flu', more than 20 million people died worldwide and countless others were hospitalized. In Canada alone, up to 50000 deaths occurred due to this pandemic (Tam 1999). The 1918 flu has become an iconic event in Canadian history, lore and policymaking. Given the history of influenza (four or five pandemics in the 19th century, three in the 20th century, and one in the first decade of the 21st century), and the mutation rate of the virus, pandemics are now considered inevitable. Yet, even without considering pandemics, 
influenza currently causes 250000 to 500000 deaths annually throughout the world (WHO 2005). Annual influenza results in great costs, in terms of death and morbidity, loss of productivity, visits to health care facilities, and hospital admissions (Szucs 1999). Influenza is therefore a matter of keen interest to policy makers in Canada and elsewhere, who must manage yearly vaccination programs, access and procedures as well as striving to use health care resources in the best ways possible. For that reason, long term trends in influenza mortality are of great interest.

The principal measure for preventing influenza and reducing the impact of epidemics is vaccination. Influenza vaccines of varying types have been available and used since after the Second World War (White \& Fenner 1994). According to the World Health Organization (WHO), vaccination is thought to reduce influenza-related hospitalizations by 25-39\% and influenza-related mortality by 39-75\% among the elderly (WHO 2005). Among healthy adults, the vaccine is very effective (70-90\% reduction in morbidity) and yields substantial health benefit (WHO 2005). Yet, influenza vaccines are typically administered to prevent severe complications due to the disease, not necessarily to control the spread of an epidemic (Fukuda \& Kieny 2006). Further, the effectiveness of the vaccine depends on variety of factors, including how well its composition of viral strains matches those circulating in the population (Claas \& Osterhaus 1998). Ultimately, vaccination strategies aimed at reducing the burden of influenza would benefit from a better understanding than we currently have of the long-term dynamics of influenza.

The influenza virus is a single-stranded RNA genome enclosed within an outer lipoprotein envelope. Protruding from this envelope are two proteins, the neuraminidase (NA) and the haemagglutinin (HA). The latter is responsible for interactions with host cells during attachment and uptake of virions. Currently, there are three genera of influenza viruses responsible for infection in humans: types A and B, which appear ubiquitously every year and are responsible for epidemics and increased rates of hospitalization, and type $C$, which shares no antigens with A or B, and which infects children frequently without causing significant disease (White \& Fenner 1994). Types B and C undergo relatively slow changes in HA over time and are only known to affect humans. In contrast, type A undergoes frequent minor, and sometimes major, changes in HA and NA and affects humans and certain non-human animals (e.g., aquatic birds, pigs, horses) (Cox \& Subbarao 2000).

\subsection{Virus and transmission}

Influenza epidemiologic dynamics remain seasonal and complex, characterized by animal reservoirs impacting and cross infecting human populations. Pandemics and epidemics often occur when influenza viruses with novel HA or NA proteins appear in the human population (Potter 2001). Novel strains may be introduced if influenza is passed directly to humans from another animal without re-assortment. Alternatively, novel strains can arise through re-assortment of the viral genome within host cells that are co-infected by two or more strains of influenza. Both processes are referred to as 'antigenic shift' (Cox \& Subbarao 2000). Evidence suggests that most influenza A pandemics in the 20th century emerged from China, where close mingling of ducks, pigs, and humans allowed viral re-assortment (Claas \& Osterhaus 1998) and transmission across the species barrier to humans. In addition, the appearance of minor mutations of HA or NA ('antigenic drift') through, for example, selective antibody pressure in infected humans, can result in localized outbreaks of influenza (Lofgren et al, 2007). Both antigenic shift and drift are thought to produce influenza subtypes that are not recognized by the human immune system, thus rendering previous immunity less effective (Claas \& Osterhaus 1998).

Since the Spanish flu, numerous smaller epidemics have been documented in North America, including the outbreaks of 1929 (Collins 1930), 1937 (the 'San Francisco Outbreak') (Greiger \& Gray 1937), and 1976 ('Swine Flu') (Gaydos et al, 2006). The within-year seasonality of influenza is well known in temperate regions (Grassly \& Fraser 2006), with peak prevalence and associated peak hospitalization occurring during the winter months. Previous studies have also examined the dynamics of this disease for uninterrupted periods of up to 30 years (Crighton et al, 2004; Groll et al, 2006; Monto et al., 1985; Viboud et al, 2006; Schanzer et al, 2007). Few studies consider long-term patterns of influenza mortality, however, and those that do often report incomplete time series (Simonsen et $a l$, 1998). Only a single study has considered the complete time series of influenza mortality in the United States since the Spanish flu (Doshi 2008); currently, there is no equivalent study on Canadian data. Accordingly, our objectives are threefold: (1) to report annual influenza mortality in Canada from 1922-2003 and compare the fit of various regression models to formally assess whether or not Canadian influenza mortality declined lawfully throughout the 82-year period; (2) compare rates of Canadian mortality ascribed to influenza and, separately, to pneumonia and all causes combined; and (3) correlate the relative abundance of any influence viral subtype and the observed mortality rate. 


\subsection{The Canadian data}

Canada began the 20th century as a sparsely populated nation of rural, urban and remote communities; she became more urbanized over the period as population growth largely affected the cities. At the end of the Century, she was relatively urban, though still somewhat sparsely populated by many other nations' standards. Data from Canada may be seen as representative of other urbanized, disparate, prosperous, uncrowded, and temperate to polar nations and her information may apply in other comparable jurisdictions, particularly insofar as they have a similar history of influenza experience.

\section{Materials and methods}

\subsection{Mortality data}

We obtained rates of Canadian mortality ascribed to influenza (1922-2003) and, separately, to pneumonia (1923-1978) and all causes combined (1923-1974), from the archives of Statistics Canada and the Canadian Institute of Health Information. Data on the circulating strains of influenza in Canada (1984-2003) were acquired from the archives of the Public Health Agency of Canada (PHAC 2007). We report on influenza deaths specifically, i.e., all deaths for which influenza was the primary cause named, regardless of other complications, using the pneumonia and all-cause time series for comparison. Although the formal delineation of influenza mortality, as given in the International Classification of Diseases (ICD), has changed several times since 1922, the available comparability statistics (the number of influenza deaths according to the previous classification versus the number according to the new classification, expressed as a ratio) indicate good agreement between successive changes in classification. Although it has been argued that the number of reported influenza deaths may underestimate the true mortality due to this disease (Schanzer 2007), there is no consensus on the best methods for estimating the true number of influenza deaths. For clarity, we therefore used the influenza specific mortality rate (per 100000 population) without adjustment or standardization.

\subsection{Statistical analyses}

We fit a variety of linear and non-linear functions to the influenza time series for the period 1922-2003 (SAS 2006). However, a simple exponential decay provided the best fit, i.e., it minimized the unexplained (error) variance of the regression model. We extracted the residuals from a linear regression of mortality rate (in transformed) against time for all further analyses.

We examined the (residual) time series for non-randomness in two ways: first, we asked if the overall time series differed statistically from white noise, i.e., if observations in the series were independent of one another, using Bartlett's Kolmogorov-Smirnov statistic (Bartlett's K-S) (SAS 2006) second, we tested for first- and higher-order autocorrelation in the residual time series using generalized Durbin-Watson statistics (SAS 2006).

We compared the fit of various regression models to formally assess whether or not Canadian influenza mortality declined smoothly, i.e., at a constant rate, throughout the period 1922-2003. We used the Joinpoint Regression Program (2010), which identifies changes in trend data using a grid-search method to fit regression functions with unknown change-points. Technical details of this approach are described elsewhere (Kim et al, 2000). The software uses either permutation tests or the Bayesian Information Criterion (BIC) to determine the best-fit model. We present BIC results for zero (constant rate of decline) to seven change-points, but note that permutation tests found the same best-fit model for our data.

\section{Results}

\subsection{Influenza}

In Canada, the annual mortality rate due to influenza declined exponentially during 1922-2003 (Fig. 1A). Whereas influenza caused, on average, 44 deaths per 100000 during the 1920s (between $\sim 1300$-7200 total deaths per year), this number dropped to only 1 per 100000 by the 1990s ( 120-760 total deaths per year). This decline corresponded to an annual percent change in influenza mortality of $-5.6 \%(95 \% \mathrm{CI}:-6.0,-5.1)$ and a linear decline in the log-transformed mortality rate (slope $=-0.057$; S.E. $=0.002$; Figure $1 \mathrm{~B}$ ). Despite this steady decline, several periods of high or low influenza mortality are apparent in our time series (Figure 1C). The years 1929, 1937, 1951, and 1976, for example, are notable for their high incidences of influenza (between 1.5-1.8 standard deviations above the long-term trend), and correspond with well documented 'outbreak years' in other parts of the world.

The interval 1998 to 2000 is notable in our time series; during these years, influenza mortality (in transformed) was 2.5-3.0 standard deviations above the decline that characterized the preceding 75 years (Figure 1). 1998 mortality was 3.0 standard deviations above the mean. While it is plausible that one result that unusual may 
show up in 82 data points, the next two years' rates repeat the anomaly.

Figure 2 shows that three primary subtypes of influenza circulated among Canadians during the ten-year period (1984-2003) surrounding this peak: types A/H1N1 and A/H3N2, and type B. Subtype A/H3N2 was clearly the most common during these ten years, and slight peaks in the relative abundance of type B bookended the mortality of 1998-2000. However, there was no clear correlation between the relative abundance of any subtype and the observed mortality due to influenza during this time (correlation coefficients, $\mathrm{A} / \mathrm{H} 1 \mathrm{~N} 1:-0.17, P=0.48$; A/H3N2: $0.07, P=0.82$; Type B: $0.08, P=0.75$ ). Hence, it does not appear that the peak in influenza mortality during 1998-2000 was simply the result of fluctuations in the circulating subtypes or the emergence of a new subtype. Time-series analyses did not show strong recurrent patterns in influenza mortality.

We analyzed the influenza series formally for any discontinuity or discontinuities to be found in the series and found only a suggestion of an uptick in 1997-98 and of a down-tick at 2000-01, This variant allows for a steeper and less noisy slope 1923 to 2007 and accounts for marginally more of the variance.

In the series, 1922-2003, mortality was similar in adjacent years (first-order autocorrelation, DW $=1.47, P=$ 0.006). However, after truncating the time series to exclude the peak during 1998-2000, influenza mortality no longer showed first-order (year-by-year) autocorrelation ( $\mathrm{DW}=2.02, P=0.49$ ), and the residuals did not differ significantly from random fluctuations (Bartlett's $\mathrm{K}-\mathrm{S}=0.12, P=0.65$ ). There was a tendency for influenza mortality to be similar every other year, i.e., alternate year-by-year, slightly high then slightly low, in the truncated time series (second-order autocorrelation, DW $=1.56, P=0.03$ ). No autocorrelations beyond second order (lag 2) approached significance.

In order to determine if significant change-points exist in the log-transformed influenza mortality data, we compared the fit of various piecewise linear regression models. Table 1 shows that the best fit model for the period 1922-2003 has two significant change-points, at 1995 and 1998, corresponding to the peak in influenza mortality during 1998-2000. This model suggests that the rate of decline was essentially constant throughout 1922-1995. Models that are more complex suggest change-points at well-known outbreak years (e.g., 1937, 1976) and during periods with unusually low rates of influenza mortality (e.g., 1965, 1979); however, these models fit less well than the simpler models.

\subsection{Pneumonia}

Pneumonia series can be described by a single decay $(-2.8 \%[-3.0,-2.6])$ but there seemed to be a discontinuity after WWII, deeper analysis suggests that and accounts again for marginally more of the variance in the residuals.

\subsection{All-cause}

Figure 3 compares Canadian mortality due to influenza, pneumonia, and all causes combined during 1923-1978. The log-transformed mortality rates declined linearly for influenza - annual percent change -6.1\% [95\% CI: -6.8, $-5.4])$ and all causes combined $-0.9 \%[-0.9,1.0])$, indicating that each of these sources of mortality decayed exponentially during the 56 year period. Influenza mortality declined significantly more quickly during this period than mortality from all causes (slope $=-0.063$; S.E. $=0.004$ vs. slope $=-0.009$; S.E. $=0.0003 ; F_{3,104}=$ 199.12, $P<0.001$ ) or from pneumonia alone (slope $=-0.063$; S.E. 0.004 vs. slope $=-0.028$; S.E. $=0.0009 ; F_{3,107}$ $=88.49, P<0.001$ ).

\section{Discussion}

\subsection{The decay}

The most easily visible aspect of influenza mortality in Canada is that, since just after the 1918 'Spanish flu' pandemic, the death rate due to this disease declined exponentially. This pattern is consistent with the decline in influenza mortality reported in the United States during the $20^{\text {th }}$ century (Doshi 2008). An exponential decay is the most parsimonious statistical model of influenza mortality in Canada since the Spanish flu. Although other models could be used to describe these data ["fit the curve"], an exponential decay requires the fewest parameters and makes the fewest assumptions in order to fit the data.

While a decay function captures the mortality rate over a lengthy period (1922-2003); there was a deviation above the predicted trend line in influenza mortality during 1998-2000. Canadian influenza mortality during 1998-2000 was clearly above the trend line that characterized the preceding 75 years. After 2001, mortality returned to near the earlier trend until 2003 where our series ends, see Fig. 1. In Canada, the 1998-2000 peak was not remarked at the time and was scarcely visible in the untransformed data; nevertheless, we show that the transformed death rate was 3.0 standard deviations above the trend line, and this was followed by two years of 
death rates $>2.5$ standard deviations above the trend line. Our preliminary examination of the strain data from that period does not suggest that this peak was the result of the appearance of a novel subtype (e.g., A/H5N1) or the result of fluctuations in the typical circulating subtypes. Although subtype A/H3N2 dominated during the entire period 1984-2003 (Fig. 2), other data shows that one particular strain, A/Sydney/05/97-like (H3N2), dominated during the peak of 1998-2000 (PHAC 2002). This strain has been implicated in other outbreaks (Uyeki et al, 2003; de Jong et al, 2000; CDC 1997) and may well be worthy of further study. It is also noteworthy that the 1997/98 influenza vaccine, which was developed based on the data available in February 1997, did not protect against A/Sydney/05/97, which emerged in Australia and New Zealand during June of 1997 (de Jong et al, 2000).

\subsection{Comparable diseases}

How do historical rates of mortality due to influenza compare with those due to other infectious respiratory diseases? Previous work shows that the mortality rate due to pulmonary tuberculosis (TB) in England and Wales during the $19^{\text {th }}$ and $20^{\text {th }}$ centuries followed an exponential decline (Wilson 1990; Blower et al, 1995). We show here that Canadian mortality due to pneumonia during the $20^{\text {th }}$ century can also be well described by an exponential decay, accounting for a little more of the variance with a stepwise discontinuity inset after WWII, as penicillin and other antibiotics came into general medical use in Canada. In North America, other infectious respiratory diseases, including diphtheria (Armstrong et al, 1999) and rheumatic fever (Shulman et al, 2006), also declined rapidly, if not exponentially, during the 1900s. Thus, it appears that the decline we observed in influenza mortality was not necessarily unique among infectious respiratory diseases during the $20^{\text {th }}$ century. What might account for such declines in mortality due to infectious respiratory diseases, and can the historical declines of TB and pneumonia in Canada shed light on the exponential decay in influenza mortality?

With respect to tuberculosis in England and Wales, historical analysis suggests that improvements in public health practice, particularly the increasingly stringent isolation of infectious individuals, contributed to the gradual decline of this disease during the late 1800s and early 1900s, whereas the use of antibiotics after WWII was the primary driver of the stark exponential drop in TB observed during the latter half of the $20^{\text {th }}$ century (Wilson 1990). Similarly, in Canada, the incidence of TB declined gradually from the mid-1920s until the late 1940s, after which the decrease became a steep exponential decline (PHAC 1998). The underlying causes of TB decline in Canada were probably very similar to those in England and Wales, with the early gradual drop resulting from general improvements in living conditions, nutrition, and a growing awareness of good public health practice, and the later exponential drop resulting from an increasing availability of antibiotics (e.g. penicillin) for the treatment of infectious respiratory diseases. However, with respect to influenza, we show that the mortality rate has been dropping at the same exponential rate since the early 1920s, without any period of gradual decline, or any evidence of acceleration during the 'antibiotic era' post-WWII. Wisconsin data from 1967 to 2004 show a linear decline in flu season duration among children and infants, and season intensities over the period appear to have declined exponentially (Lofgren et al, 2010).

Although improved public health practice and changes in antibiotic availability may have contributed to a general decrease in infectious respiratory diseases in North America (Armstrong et al, 1999), particularly with regard to heaths from pneumonia and $\mathrm{TB}$, these factors by themselves do not adequately explain the lawful exponential decay of influenza deaths in Canada.

Pneumonia is a common complication arising during influenza infection. Historically, many believe that many deaths that began as influenza illness may have been ascribed to pneumonia. Indeed, due to this ambiguity, some researchers have simply grouped influenza and pneumonia deaths together ('P\&I') when analyzing historical patterns (Viboud et al, 2006; Simonsen et al, 1998). Could such a classification artefact account for the apparent decline in influenza deaths? This seems unlikely, given that influenza and pneumonia death rates have both declined exponentially in Canada since the early 1900s (see Fig. 3). There has been a slower but steady decay in all-cause mortality over the same time.

Plotting mortality ascribed to all infectious respiratory diseases, other than TB, in Ontario from 1880 to1930 on semi-log paper, Ross (1935) showed a decreasing pattern before 1918, a spike in 1918 [the Spanish flu] and decreasing after that peak. The decreasing periods both fit an exponential law. This has two impacts on interpreting the present dataset work-it shows that deaths from all respiratory diseases combined (including flu) seemed to decline in the period after 1922, though it declined more slowly than influenza. It raises the question, was 1918 a singular event from which the death rate regressed back to the baseline trend? 


\subsection{Data quality}

Data validation: Canadian influenza death rate data are official, collected by a national professional statistical agency, making every effort to assure that methodologies are comparable, so far as is possible, year over year and decade over decade. As these are historic records, no further validation seems feasible.

Notwithstanding any of the foregoing, data quality remains an important issue in influenza research. Some studies estimate that, due to the absence of routine diagnosis, the actual number of influenza-attributable deaths may be ten times higher than reported (Schanzer et al, 2007). Although it must be true that there are more influenza deaths than are reported, this cannot be responsible for the historical decline in influenza-related mortality unless reporting practices have become progressively worse since the Spanish flu. Note that there may have been systematic tendency not to record deaths due to influenza that should have been so classified, for example, cases that ended with pneumonia as the proximate cause of death may have began as 'flu; 'flu can also be a significant event in the causal chain leading to death from heart disease. Indeed we expect there has been a great deal of flu death applied to other causes. But such systematic variation in naming deaths as due to other causes would be expected to this would show up as steps or bends in the re-expressed data series at the points of changed terminology. As steps or bends were not seen in the broad sweep of the 'flu data, at least until 1998, we had no reason to assume that the broad proportions of labeling/mislabeling had varied over the study period; it was most parsimonious to make no such assumption and we did not make any. This has the effect of assuming constant mislabeling proportions over the period but that is the most parsimonious alternative available. As the spread of the data points around the trend line is also normalized by the log transform, we see no reason to make any more complex hypothesis.

The coding of influenza-related deaths according to the International Classification of Diseases (ICD) [http://www.wolfbane.com/icd/] has changed on several occasions during our time series (i.e., during 1930, 1939, 1949, 1958, 1968, 1979, and 1999). If influenza became a less frequent cause of attributed death due to changes in the ICD naming conventions, sudden decreases ('steps') should appear in the influenza time series during those years. However, we see no evidence of this in our data. Influenza was not mentioned as a cause of death in conjunction with any other disease until ICD-5 was introduced in 1938, wherein influenza was noted as a cause of disease and as a contributing factor to deaths by pneumonia and asthma. ICD-6, in 1948, distinguished 'influenza', 'influenza with pneumonia', 'influenza unqualified or with other respiratory complications', 'influenza with digestive complications', and 'influenza with nervous system involvement'. This situation remained unchanged in ICD-7 and virtually unchanged in ICD-8, except that influenza was expressly separated from acute respiratory infections other than pneumonia. ICD-9 in 1975 expressly separated all forms of pneumonia from influenza, and ICD-10 in 1990 separated influenza, pneumonia with influenza virus, and other pneumonias. These changes did not impact the level or rate of the observed decay in influenza mortality. Indeed, there was no apparent change in the observed trend from any change in medical practice or diagnosis. If improvements in medicine or diagnosis were responsible for the long-term decline, such improvements would need to have been progressive and multiplicative year-over-year to account for the data, which seems unlikely.

\subsection{Immunization Programs}

Could the decline of influenza also be due to immunization programs? Despite the fact that influenza vaccines have been available since the 1940s, widespread use of the 'flu shot' did not occur in Canada until the 1990s (Fedson 1995; Kwong et al, 2008). Hence, it is unlikely that vaccination programs were responsible for the large declines observed in Canadian influenza mortality during the early and mid-20 $0^{\text {th }}$ century. Nevertheless, later analysis in Ontario indicates that vaccination programs have been effective in recent years.

Exposure during seasonal influenza outbreaks, or through vaccination, may provide individuals with substantial cross-protection among influenza subtypes (Gioia et al, 2008; Kreijtz et al, 2008; Rimmelzwaan et al, 2007; Kreijtz et al, 2007), so-called 'memory immunity' (Ahmed et al, 2007). In addition, recent work shows that individuals can harbour highly specific immunity to an influenza strain, even 90 years after exposure (Yu et al, 2008). These mechanisms might gradually enhance a population's immunity to common influenza viruses. Indeed, other authors have remarked that the commonly circulating subtypes of influenza appear to be gradually losing their ability to cause serious disease in the humans (Webby \& Webster 2003). Further, it has been suggested that declines in influenza mortality in the United States during the $20^{\text {th }}$ century may be due to immunity acquired through exposure to circulating strains (Doshi 2008). Such enhancement of natural immunity may inform strategies that relate to vaccines and vaccination programs. For example, if exposure to (inactivated) influenza through vaccination facilitates the development of cross-protection against novel strains or subtypes, vaccinating a population with any flu vaccine likely is better than no vaccination at all. 
Future 'flu mortality: How might the observed decay in influenza mortality affect future death rates as the Canadian population ages? Unpublished analyses on age-specific death rates (Lubarsky 2004, personal communication) suggest that normal-year [non-pandemic] flu will continue to take a disproportionate toll among the elderly [70 and over], as more and more Canadians now survive to 80 years and over. An interesting point for further study is whether or not surviving any form of influenza, pandemic or not, in early life confers added immunity in later life, and whether such immunity continues to protect even beyond age 70 .

\section{Conclusion}

Analysis of Canadian influenza mortality data, since just after the 1918 'Spanish flu' pandemic, indicates that the death rate due to this disease has declined exponentially, but that the decline we observed in influenza mortality is not necessarily unique among infectious respiratory diseases during the $20^{\text {th }}$ century. Improvements in public health practices, vaccination programs and increased antibiotic availability alone do not adequately explain the lawful exponential decay of influenza deaths in Canada. Our results suggest that surviving any form of influenza confers added immunity in later life; by extension, we infer that vaccinating a population with any flu vaccine likely is better than no vaccination at all. Whether such immunity continues to protect individuals beyond age 70 is not clear; however, further comparative studies in other countries would prove useful in validating the conclusions put forth in this investigation.

\section{Acknowledgements}

We thank Brian Winchester, Public Health Agency of Canada, for valuable assistance in identifying the data. We further thank editors and referees for interesting and useful commentary.

\section{References}

Ahmed, R., Oldstone, M. B. A., \& Palese, P. (2007). Protective immunity and susceptibility to infectious diseases: lessons from the 1918 influenza pandemic. Nature, 8 (11), 1188-1193.

Armstrong, G. L., Conn, L. A., Pinner, R. W. (1999). Trends in infectious disease mortality in the United States during the 20th century. Journal of the American Medical Association, 281 (1), 61-66. http://dx.doi.org/10.1001/jama.281.1.61

Blower, S. M., McLean, A. R., Porco, T. C., Small, P. M., Hopewell, P. C., Sanchez, M. A., \& Moss, A. R. (1995). The intrinsic transmission dynamics of tuberculosis epidemics. Nature Medicine, 815-821. http://dx.doi.org/10.1038/nm0895-815

Center for Disease Control. (CDC). (1997). Update: Influenza activity-United States, 1997-98 Seasons. Morbidity and Mortality Weekly Report, 46 (46), 1094-1098.

Claas, E. C. J. \& Osterhaus, A. (1998). New clues to the emergence of flu pandemics. Nature Medicine, 4 (10), 1122-1123. http://dx.doi.org/10.1038/2617

Collins, S. D. (1930). The influenza epidemic of 1928-1929 with comparative data for 1918-1919. American Journal of Public Health, 20 (2), 119-129. http://dx.doi.org/10.2105/AJPH.20.2.119

Crighton, E. J., Moineddin, R., Mamdani, M., \& Upshur, R. E. G. (2004). Influenza and pneumonia hospitalizations in Ontario: a time-series analysis. Epidemiology and Infection, 132 (6), 1167-1174. http://dx.doi.org/10.1017/S0950268804002924

Crighton, E. J., Elliott, S. J., Moineddin, R., Kanaroglou, P., \& Upshur, R. (2007). A spatial analysis of the determinants of pneumonia and influenza hospitalizations in Ontario (1992-2001). Social Science \& Medicine, 64 (8), 1636-1650. http://dx.doi.org/10.1016/j.socscimed.2006.12.001

Cox, N. J., Subbarao, K. (2000). Global epidemiology of influenza: Past and present. Annual Review of Medicine, 51, 407-421. http://dx.doi.org/10.1146/annurev.med.51.1.407

De Jong, J. C., Beyer, W. E. P., Palache, A. M., Rimmelzwaan, G. F., \& Osterhaus, A. (2000). Mismatch between the 1997/1998 influenza vaccine and the major epidemic A (H3N2) virus strain as the cause of an inadequate vaccine-induced antibody response to this strain in the elderly. Journal of Medical Virology, 61 (1), 94-99. http://dx.doi.org/10.1002/(SICI)1096-9071(200005)61:1<94::AID-JMV15>3.0.CO;2-C

Doshi, P. (2008). Trends in recorded influenza mortality: United States, 1900-2004. American Journal of Public Health, 98 (5), 939-945. http://dx.doi.org/10.2105/AJPH.2007.119933

Fedson, D. S. (1995). Influenza and pneumococcal vaccination in Canada and the United-States, 1980-1993: What can the 2 countries learn from each other? Clinical Infectious Diseases, 20 (5), 1371-1376. http://dx.doi.org/10.1093/clinids/20.5.1371 
Fukuda, K. \& Kieny, M. P. (2006). Different approaches to influenza vaccination. New England Journal of Medicine, 355 (24), 2586-2587. http://dx.doi.org/10.1056/NEJMe068257

Gaydos, J. C., Top, F. H., Hodder, R. A., \& Russell, P. K. (2006). Swine influenza a outbreak, Fort Dix, New Jersey, 1976. Emerging Infectious Diseases, 12 (1), 23-28.

Gioia, C., Castilletti, C., Tempestilli, M., Piacentini, P., Bordi, L., \& Chiappini, R. (2008). Cross-subtype immunity against avian influenza in-persons recently vaccinated for influenza. Emerging Infectious Diseases, 14, 121-128. http://dx.doi.org/10.3201/eid1401.061283

Geiger, J. C. \& Gray, J. P. (1937). Influenza: the San Francisco outbreak of January-February, 1937. California and Western Medicine, 46 (6), 379-381.

Grassly, N. C. \& Fraser, C. (2006). Seasonal infectious disease epidemiology. Proceedings of the Royal Society B-Biological Sciences, 273 (1600), 2541-2550. http://dx.doi.org/10.1098/rspb.2006.3604

Groll, D. L. \& Thomson, D. J. (2006). Incidence of influenza in Ontario following the Universal Influenza Immunization Campaign. Vaccine, 24 (24), 5245-5250. http://dx.doi.org/10.1016/j.vaccine.2006.03.067

Joinpoint Regression Program, Version 3.4.3. (2010). Statistical Research and Applications Branch. National Cancer Institute.

Kim, H. J. Fay, M. P. Feuer, E. J., \& Midthune, D. N. (2001). Permutation tests for joinpoint regression with applications to cancer rates. Statistics in Medicine, 20, 655. http://dx.doi.org/10.1002/sim.811

Kreijtz, J., Bodewes, R., van Amerongen, G., Kuiken, T., Fouchier, R. A. M., \& Osterhaus, A. (2007). Primary influenza a virus infection induces cross-protective immunity against a lethal infection with a heterosubtypic virus strain in mice. Vaccine, 25 (4), 612-620. http://dx.doi.org/10.1016/j.vaccine.2006.08.036

Kreijtz, J., de Mutsert, G., van Baalen, C. A., Fouchier, R. A. M., Osterhaus, A., \& Rimmelzwaan, G. F. (2008). Cross-recognition of avian H5N1 influenza virus by human cytotoxic T-lymphocyte populations directed to human influenza a virus. Journal of Virology, 82 (11), 5161-5166. http://dx.doi.org/10.1128/JVI.02694-07

Kwong, J. C., Stukel, T. A., Lim, J., McGeer, A. J., Upshur, R. E. G., \& Johansen, H. (2008). The effect of Universal Influenza Immunization on mortality and health care use. Plos Medicine, 5 (10), 1440-1452. http://dx.doi.org/10.1371/journal.pmed.0050211

Lofgren, E., Fefferman, N. H., Naumov, Y. N., Gorski, J., \& Naumova, E. N. (2007). Influenza seasonality: Underlying causes and modeling theories. Journal of Virology, 81 (11), 5429-5436. http://dx.doi.org/10.1128/JVI.01680-06

Lofgren, E. T., Wenger, J. B., Fefferman, N. H., Bina, D., Gradus, S., Bhattacharyya, S., Naumov, Y. N., Gorski, J., \& Naumova, E. N. (2010). Disproportional effects in populations of concern for pandemic influenza: insights from seasonal epidemics in Wisconsin 1967-2004. Influenza and Other Respiratory Viruses, 4, 205-212. http://dx.doi.org/10.1111/j.1750-2659.2010.00137.x

Monto, A. S., Koopman, J. S., \& Longini, I. M. (1985). Tecumseh Study of Illness. XIII. Influenza Infection and Disease, 1976-1981. American Journal of Epidemiology, 121 (6), 811-822.

Potter, C. W. (2001). A history of influenza. Journal of Applied Microbiology, 91 (4), 572-579. http://dx.doi.org/10.1046/j.1365-2672.2001.01492.x

Public Health Agency of Canada (PHAC). (1998). Tuberculosis in Canada. [Online] Available: http://www.phac-aspc.gc.ca/publicat/tbcan98/index-eng.php.

Public Health Agency of Canada (PHAC). (1998). Guidelines for the control of diphtheria in Canada. Canada Communicable Disease Report, 24S3.

Public Health Agency of Canada (PHAC). (2002). Influenza in Canada: 2000-2001 season. Public Health Agency of Canada, February 1.

Public Health Agency of Canada (PHAC). (2007). Annual \& Interim Influenza Surveillance Reports. Public Health Agency of Canada.

Rimmelzwaan, G. F., Fouchier, R. A. M., \& Osterhaus, A. (2007). Influenza virus-specific cytotoxic T lymphocytes: a correlate of protection and a basis for vaccine development. Current Opinion in Biotechnology, 18 (6), 529-536. http://dx.doi.org/10.1016/j.copbio.2007.11.002

Ross, M. A. (1935). Mortality from respiratory diseases excluding tuberculosis: Ontario 1880-1931. Canadian Public Health Journal, 26. 
SAS Institute. (2006). SAS/STAT 9.1 User's Guide. Cary: SAS Institute.

Schanzer, D. L., Tam, T. W. S., Langlev, J. M., \& Winchester, B. T. (2007). Influenza-attributable deaths, Canada 1990-1999. Epidemiology and Infection, 135 (7), 1109-1116. http://dx.doi.org/10.1017/S0950268807007923

Shulman, S. T., Stollerman, G., Beall, B., Dale, J. B., \& Tanz, R. R. (2006). Temporal changes in streptococcal M protein types and the near-disappearance of acute rheumatic fever in the United States. Clinical Infectious Diseases, 42, 441-447. http://dx.doi.org/10.1086/499812

Simonsen, L., Clarke, M. J., Schonberger, L. B., Arden, N. H., Cox, N. J., \& Fukuda, K. (1998). Pandemic versus epidemic influenza mortality: A pattern of changing age distribution. Journal of Infectious Diseases, 178 (1), 53-60.

Szucs, T. (1999). The socio-economic burden of influenza. Journal of Antimicrobial Chemotherapy. 44, 11-14. http://dx.doi.org/10.1093/jac/44.suppl_2.11

Tam, T. W. S. (1999). Preparing for influenza epidemics and pandemics in the new millennium. Canadian Journal of Public Health-Revue Canadienne De Sante Publique, 90 (5), 293-295.

Uyeki, T. M., Zane, S. B., Bodnar, U. R., Fielding, K. L., Buxton, J. A., \& Miller, J. M. (2003). Large summertime influenza a outbreak among tourists in Alaska and the Yukon Territory. Clinical Infectious Diseases, 36 (9), 1095-1102. http://dx.doi.org/10.1086/374053

Viboud, C., Bjørnstad, O. N., Smith, D. L., Simonsen, L., Miller, M. A., \& Grenfell, B. T. (2006). Synchrony, waves, and spatial hierarchies in the spread of influenza. Science, 312 (5772), 447-451. http://dx.doi.org/10.1126/science.1125237

Webby, R. J. \& Webster, R. G. (2003). Are we ready for pandemic influenza? Science, 302 (5650), 1519-1522. http://dx.doi.org/10.1126/science.1090350

White, D. O. \& Fenner, F. J. (1994). Medical Virology (4th. Ed.). San Diego: Academic Press.

Wilson, L. G. (1990). The historical decline of tuberculosis in Europe and America: Its causes on significance. Journal of the History of Medicine, 45, 366-396.

World Health Organization (WHO). (2005). Weekly Epidemiological Record, 80 (33), 277-288.

Yu, X. C., Tsibane, T., McGraw, P. A., House, F. S., Keefer, C. J., \& Hicar, M. D. (2008). Neutralizing antibodies derived from the B cells of 1918 influenza pandemic survivors. Nature, 455 (7212), 532-541. http://dx.doi.org/10.1038/nature07231

Table 1. Statistics describing the fit of various piecewise linear regression models to the Canadian influenza mortality rate, 1922-2003. The model with the lowest Bayesian Information Criterion (BIC) value, shown in bold, is deemed the best fit

\begin{tabular}{|c|l|c|c|c|}
\hline $\begin{array}{c}\text { Number of } \\
\text { change-points }\end{array}$ & \multicolumn{1}{|c|}{ Position of change-points } & $\begin{array}{c}\text { Number of model } \\
\text { parameters }\end{array}$ & df & BIC \\
\hline 0 & - & 2 & 80 & -1.30 \\
\hline 1 & 1989 & 4 & 78 & -1.42 \\
\hline $\mathbf{2}$ & $\mathbf{1 9 9 5 , \mathbf { 1 9 9 8 }}$ & $\mathbf{6}$ & $\mathbf{7 6}$ & $\mathbf{- 1 . 4 6}$ \\
\hline 3 & $1995,1998,2001$ & 8 & 74 & -1.39 \\
\hline 4 & $1939,1947,1995,1998$ & 10 & 72 & -1.35 \\
\hline 5 & $1939,1947,1995,1998,2001$ & 12 & 70 & -1.28 \\
\hline 6 & $1937,1965,1976,1979,1995,1998$ & 14 & 68 & -1.23 \\
\hline 7 & $1937,1965,1976,1979,1995,1998,2001$ & 16 & 66 & -1.16 \\
\hline
\end{tabular}



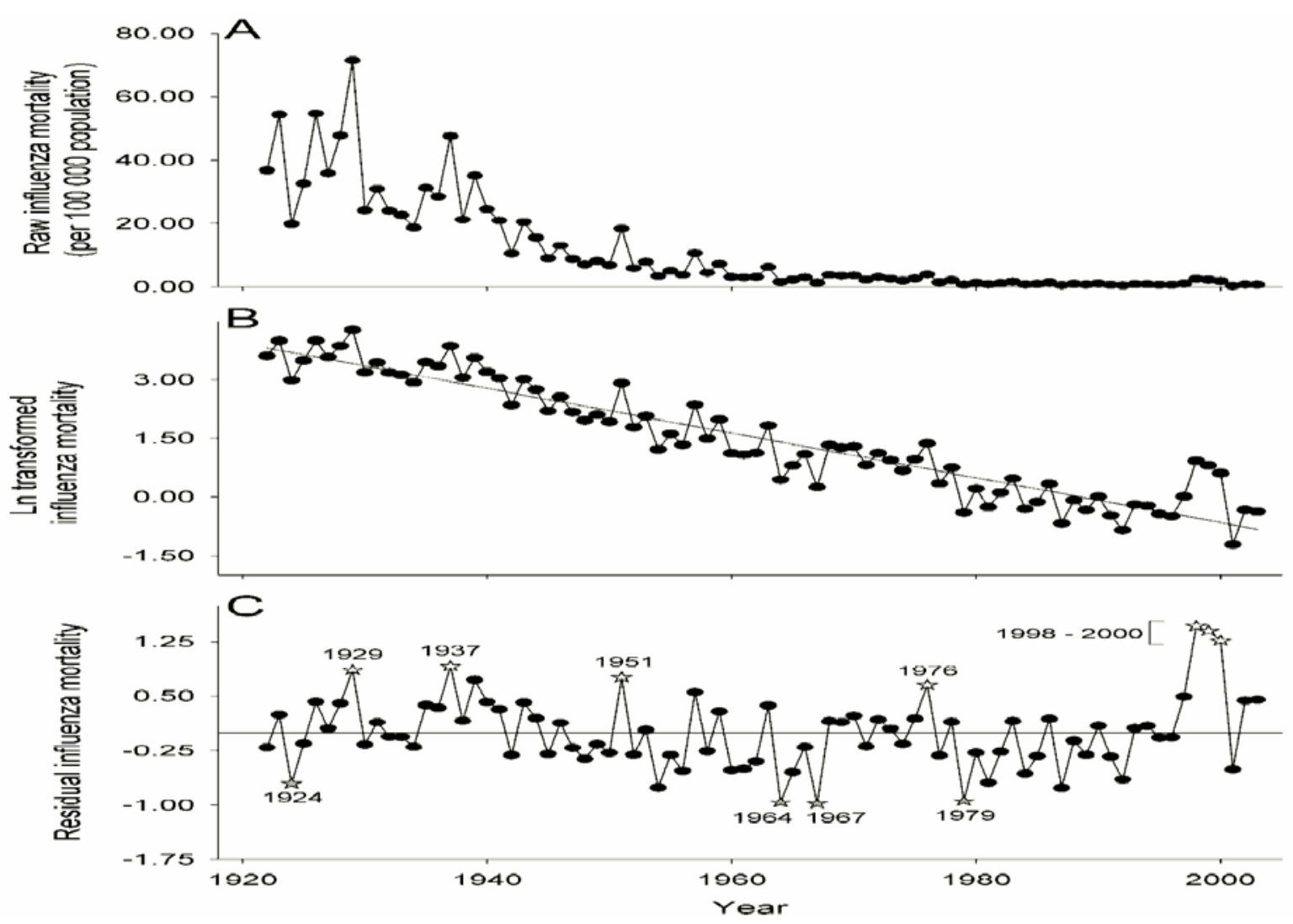

Figure 1. Annual Canadian mortality rate due to influenza during 1922-2003

Panel A shows the influenza specific mortality rate (per 100 000) due to influenza; panel B shows the influenza specific mortality rate, natural $\log$ transformed, with a solid line indicating the linear regression fit, $\mathrm{y}=-0.057 \mathrm{x}+$ $113.73\left(F_{1,80}=596.99, P<0.001, R^{2}=0.88\right)$; panel C shows the residual influenza mortality (error terms from the linear regression of transformed mortality on year), with mortality values approximately 1.5-3.0 S.D. above the long-term trend and mortality values approximately 1.5-2.0 S.D. below the long-term trend (indicated by open and filled stars, respectively).

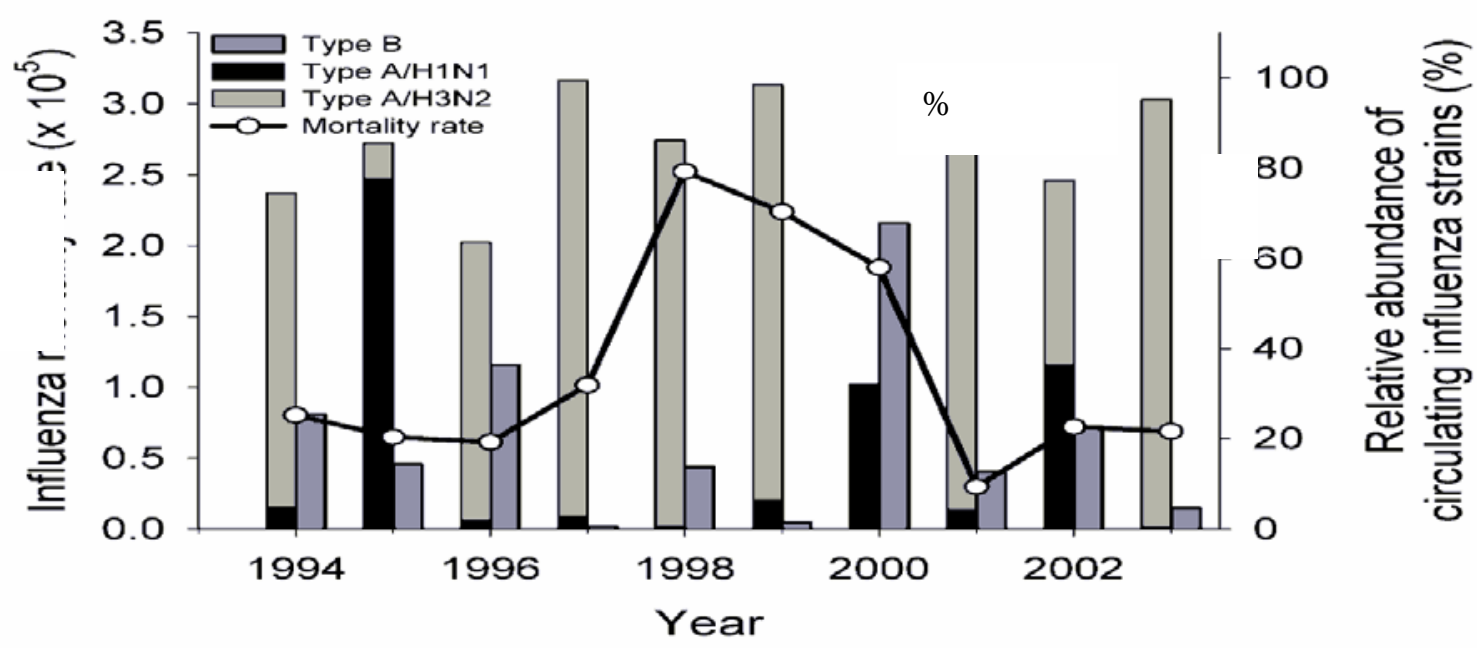

Figure 2. Frequency histograms show the relative abundance of the various influenza strains that circulated in Canada during 1994-2003

A peak in influenza mortality observed in Canada during 1998-2000; for comparison, the influenza-specific mortality rates are shown for the period 1994-2003 


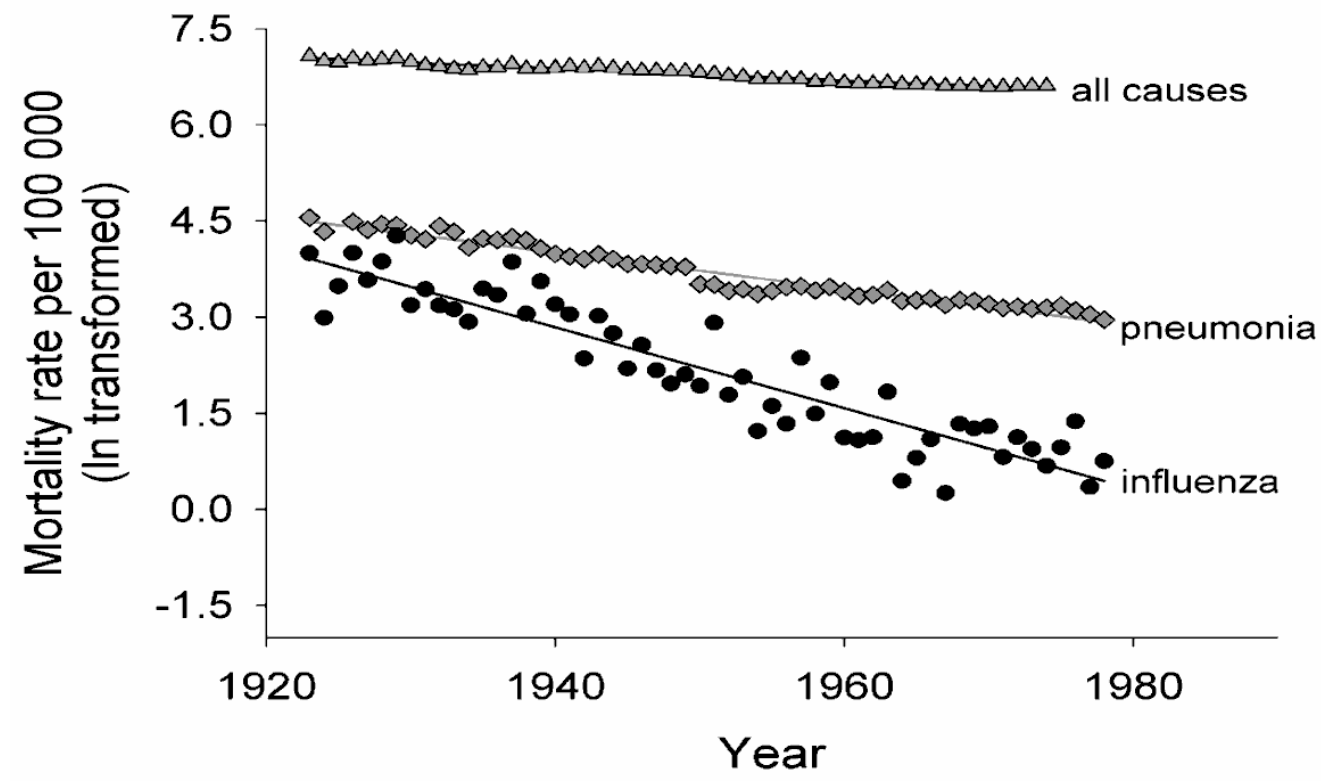

Figure 3. A comparison of annual Canadian mortality rates from all causes combined, pneumonia only, and influenza only, for the period 1923-1978

Note that the regression equations are as follows:

Influenza: $\operatorname{Ln}($ rate $)=-0.063$ (year) +125.179

Pneumonia: $\operatorname{Ln}($ rate $)=-0.028$ (year $)+59.292$

All Cause: $\operatorname{Ln}($ rate $)=-0.009$ (year) +25.034 